Princeton, United States of America; ${ }^{9}$ Bristol Myers Squibb, Rheumatology and Dermatology, Princeton, United States of America

Background: Tyrosine kinase 2 (TYK2) is an intracellular kinase that mediates signaling by key cytokines involved in psoriatic arthritis (PsA) and plaque psoriasis $(\mathrm{PsO})$ pathogenesis. Deucravacitinib is a novel oral agent that selectively inhibits TYK2 via an allosteric mechanism by binding to the nonconserved regulatory domain of the kinase. A previous Phase 2 trial in $\mathrm{PsO}$ had demonstrated that deucravacitinib was efficacious and well tolerated, with no laboratory abnormalities observed

Objectives: To evaluate the efficacy and safety of deucravacitinib in active PsA Methods: This is an ongoing, 1-year, randomized, double-blind, placebo (PBO)-controlled (initial 16 weeks), multiregional, Phase 2 trial (NCT03881059). Eligible patients had a PsA diagnosis for $\geq 6$ months, met CASPAR criteria, and had active disease with $\geq 3$ tender and $\geq 3$ swollen joints, C-reactive protein $\geq 3 \mathrm{mg} / \mathrm{L}$ (ULN, $5 \mathrm{mg} / \mathrm{L})$, and $\geq 1$ psoriatic lesion $(\geq 2 \mathrm{~cm})$. Patients had failed or were intolerant to $\geq 1$ nonsteroidal anti-inflammatory drug, corticosteroid, conventional synthetic disease-modifying antirheumatic drug (csDMARD), and/or 1 TNF inhibitor (TNFi; $\leq 30 \%$ ). Patients were randomized 1:1:1 to deucravacitinib $6 \mathrm{mg}$ once daily (QD) or $12 \mathrm{mg} Q \mathrm{QD}$, or PBO. The primary endpoint was achievement of ACR 20 response at Week 16. Additional endpoints included the proportion of patients achieving ACR 50/70 response, Health Assessment Questionnaire-Disability Index (HAQ-DI) response ( $\geq 0.35$ improvement from baseline), enthesitis resolution (Leeds Index score of 0), minimal disease activity, change from baseline in SF-36 physical component score (SF-36 PCS) and mental component score (SF-36 MCS), Psoriasis Area and Severity Index (PASI) 75 response, adverse events (AEs), and laboratory parameters.

Results: Of 203 patients randomized, 180 (89\%) completed 16 weeks of treatment (deucravacitinib $6 \mathrm{mg}$ QD, 63/70 [90\%]; deucravacitinib $12 \mathrm{mg}$ QD, 59/67 [88\%]; PBO, 58/66 [88\%]). Demographic and baseline disease characteristics were similar across groups. Mean age was 49.8 years, $51 \%$ of patients were female, median PsA duration was 4.5 years, $66 \%$ of patients used csDMARDs at baseline and throughout the study, and $15 \%$ had used a TNFi. This study met its primary endpoint, with deucravacitinib $6 \mathrm{mg}$ and $12 \mathrm{mg}$ QD demonstrating significantly higher ACR 20 responses versus PBO at Week 16 (Figure 1). Additional endpoints were also met with deucravacitinib versus PBO (Figure 1). Adjusted mean changes from baseline in SF-36 PCS and SF-36 MCS at Week 16 , respectively, were significantly higher in the deucravacitinib $6 \mathrm{mg}$ QD group (5.6 vs 2.3, $P=0.0062 ; 3.6$ vs $0.7, P=0.0211$ ) and $12 \mathrm{mg}$ QD group (5.8 vs 2.3 , $P=0.0042 ; 3.5$ vs $0.7, P=0.0263$ ) compared with $\mathrm{PBO}$. PASI 75 responses were also significantly higher in the deucravacitinib groups ( $P \leq 0.0136$ vs $P B O)$. The most common AEs in the deucravacitinib $6 \mathrm{mg} / 12 \mathrm{mg} / \mathrm{PBO}$ groups, respectively, during the 16-week treatment period were nasopharyngitis $(5.7 \% / 17.9 \% / 7.6 \%)$, sinusitis $(0 \% / 7.5 \% / 0 \%)$, headache $(7.1 \% / 1.5 \% / 4.5 \%)$, and rash $(4.3 \% / 6.0 \% / 0 \%)$. No serious AEs, herpes zoster infections, opportunistic infections, or thrombotic events were reported in deucravacitinib-treated patients during this period. Additionally, no significant changes from baseline in hematologic parameters (lymphocytes, neutrophils, platelets, and hemoglobin) or serum lipids were observed with deucravacitinib treatment.

Conclusion: Deucravacitinib was efficacious versus PBO over 16 weeks in patients with active PsA. Treatment was generally well tolerated and the safety and laboratory parameter profile of deucravacitinib was consistent with that observed in an earlier Phase $2 \mathrm{PsO}$ trial.

Acknowledgements: This study was sponsored by Bristol Myers Squibb. Professional medical writing assistance was provided by Peloton Advantage, LLC, an OPEN Health company, and funded by Bristol Myers Squibb.

Figure. Response Endpoints at Week 16

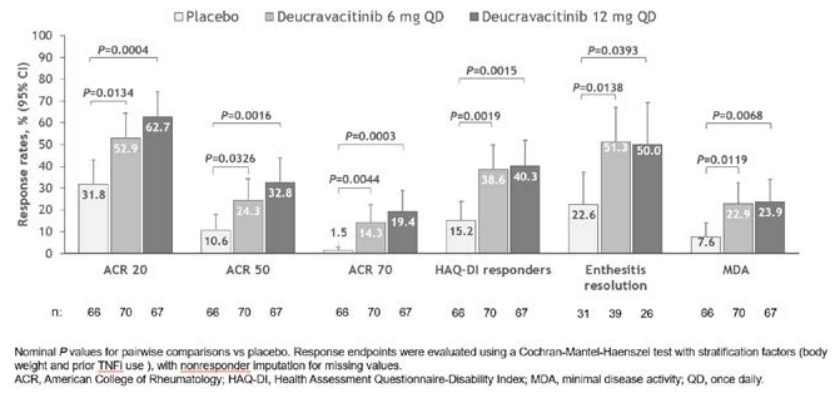

Disclosure of Interests: Philip J Mease Consultant of: AbbVie, Amgen, Boehringer Ingelheim, Bristol Myers Squibb, Eli Lilly, Galapagos, Gilead, GlaxoSmithKline, Janssen, Novartis, Pfizer, SUN Pharma, UCB, Grant/research support from: AbbVie, Amgen, Boehringer Ingelheim, Bristol Myers Squibb, Eli Lilly, Galapagos, Gilead, GlaxoSmithKline, Janssen, Novartis, Pfizer, SUN Pharma UCB, Atul Deodhar Consultant of: AbbVie, Amgen, Boehringer Ingelheim, Bris tol Myers Squibb, Celgene, Eli Lilly, Galapagos, Glaxo Smith \& Kline, Janssen Novartis, Pfizer, UCB, Grant/research support from: AbbVie, Eli Lilly, Glaxo Smith \& Kline, Novartis, Pfizer, UCB, Désirée van der Heijde Consultant of: AbbVie Amgen, Astellas, AstraZeneca, Bayer, Bristol Myers Squibb, Boehringer Ingelheim, Celgene, Cyxone, Daiichi, Eisai, Eli Lilly, Galapagos, Gilead, GlaxoSmithKline, Janssen, Merck, Novartis, Pfizer, Regeneron, Roche, Sanofi, Takeda, UCB Pharma, Frank Behrens Consultant of: Pfizer, AbbVie, Sanofi, Lilly, Novartis, Genzyme, Boehringer, Janssen, MSD, Celgene, Roche, Chugai, Bristol Myers Squibb, UCB Pharma, Grant/research support from: Pfizer, Janssen, Chugai, Celgene, Roche, Alan Kivitz Shareholder of: Pfizer, Sanofi, GlaxoSmithKline Gilead Sciences, Inc., Novartis, Paid Consultant: AbbVie, Boehringer Ingelheim, Flexion, Janssen, Pfizer, Sanofi, Regeneron, SUN Pharma Advanced Research, Gilead Sciences, Inc., Speakers bureau: Celgene, Merck, Lilly, Novartis, Pfizer, Sanofi, Genzyme, Flexion, AbbVie, Jonghyeon Kim Shareholder of: Bristol Myers Squibb, Employee of: Bristol Myers Squibb, Shalabh Singhal Shareholder of: Bristol Myers Squibb, Employee of: Bristol Myers Squibb, Miroslawa Nowak Shareholder of: Bristol Myers Squibb, Employee of: Bristol Myers Squibb, Subhashis Banerjee Shareholder of: Bristol Myers Squibb, Employee of: Bristol Myers Squibb

DOI: 10.1136/annrheumdis-2021-eular.2603

\section{POS0199 \\ TIME-SERIES ANALYSIS IN MODERATE TO SEVERE PLAQUE PSORIASIS UNDER DIFFERENT BIOLOGICS TREATMENTS}

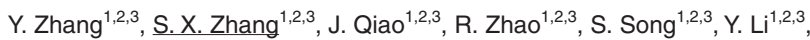
M. J. Chang ${ }^{1,2,3}$, G. Y. Liu ${ }^{1}$, P. F. He $e^{4}$, X. Li ${ }^{1,2,3}{ }^{1}$ The Second Hospital of Shanxi Medical University, Department of Rheumatology, Taiyuan, China; ${ }^{2}$ Shanxi Li Xiaofeng Medical Groups, Department of Rheumatology, Taiyuan, China; ${ }^{3}$ Ministry of Education, Key laboratory of Cellular Physiology at Shanxi Medical University, Taiyuan, China; ${ }^{4}$ Shanxi Medical University, Medical Data Sciences, Taiyuan, China

Background: Moderate to Severe Plaque Psoriasis is an inflammatory skin disease that is associated with multiple comorbidities and substantially diminishes patients' quality of life. As one of the most significant therapeutic advancements in the field of dermatology, Biologics such as TNF inhibitors, IL-12/23 inhibitor, IL-17 inhibitors, and IL-23 inhibitors, have higher efficacy compared with oral medications or phototherapy ${ }^{1}$. However, the previous studies did not focus on the simultaneous comparison of molecular changes in different classes of biologics. The identification of time-series genes (TSGs) could help to uncover the mechanisms underlying transcriptional regulation ${ }^{2}$.

Objectives: In this study, we aimed to compare the differences in expression patterns and functions of time-series genes in Moderate to Severe Plaque Psoriasis under different biologics treatments.

Methods: The transcription profile of GSE117239 and GSE51440 were obtained from the Gene Expression Omnibus database (GEO). The GSE117239 included 19 samples treated with Etanercept (TNF inhibitors) and 16 samples treated with Ustekinumab (IL-12/23 inhibitor). The GSE51440 included 4 samples treated with Guselkumab (IL-23 inhibitors). Skin biopsy samples (LS: lesion, NL: non-lesion) were collected at baseline, weeks 1 and 12, respectively. After background adjustment and other pre-procession, differentially expressed genes (DEGs) were extracted from LS skin biopsy and untreated NL skin biopsy at different times after three different biologics treatments, respectively. The Short Time-series Expression Miner (STEM) software was used to cluster and compare average DEGs with coherent changes. Afterward, the different expression patterns of TSGs under the three treatment groups were compared. GO analysis and KEGG pathway enrichment analysis of TSGs were performed by Metascape.

Results: Different DEGs varied in LS skin compared with those of NL skin biopsy: 976 genes in Ustekinumab group, 996 genes in Etanercept group, and 601 genes in Guselkumab group detailly $(P<0.05$ and [log FC] $>1)$. Gene landscapes suggested the signatures of LS gradually changed during the treatment process, and gradually converge to NL signatures (Fig.1a, 2a,3a) Time-series genes in the three treatment groups had different expression patterns and functions. In the Ustekinumab group, a total of 448 TSGs in profile 3 showed a stable-stable-decreasing expression trend and significantly 
associated with mitotic nuclear division and defense response to other organism, whereas in profile 4 represented a stable-stable-increasing expression trend and significantly associated with positive regulation of cellular response to organic 9 compound (Fig.1). With the treatment of Etanercept, 22 TSGs had a stable-increasing-increasing expression tendency and closely associated with fatty acid metabolism and steroid metabolic process (Fig.2). After Guselkumab treatment, 13 TSGs also represented a stable-increasing-increasing expression tendency that mainly characterized by defense response to other organism and epidermis development (Fig.3). Interestingly, both Ustekinumab and Guselkumab treatment dramatically influenced defense response to other organism-related genes, while Etanercept mainly affected genes involved in fatty acid metabolism and steroid metabolic process.

Conclusion: Biologics effectively reconstituted the gene signatures of psoriasis in different aspects. TSG features could be one of indicator for precise intervention for psoriasis.

\section{REFERENCES:}

[1] Armstrong AW, Read C. Pathophysiology, Clinical Presentation, and Treatment of Psoriasis: A Review. Jama 2020;323(19):1945-60. doi: 10.1001/ jama.2020.4006 [published Online First: 2020/05/20]

[2] Ernst J, Bar-Joseph Z. STEM: a tool for the analysis of short time series gene expression data. BMC Bioinformatics 2006;7:191. doi: 10.1186/1471-2105-7191 [published Online First: 2006/04/07]

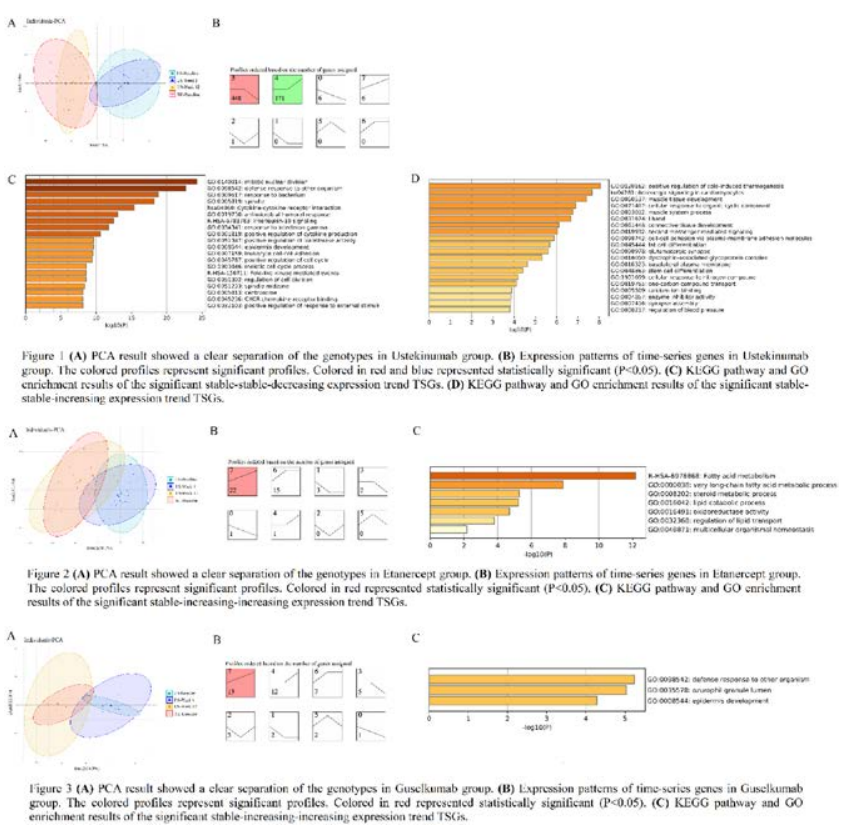

Acknowledgements: This project was supported by National Science Foundation of China (82001740), Open Fund from the Key Laboratory of Cellular Physiology (Shanxi Medical University) (KLCP2019) and Innovation Plan for Postgraduate Education in Shanxi Province (2020BY078).

Disclosure of Interests: None declared

DOI: 10.1136/annrheumdis-2021-eular.2669

\section{POS0200 1 CLINICAL CHARACTERISTICS \& OUTCOMES ASSOCIATE WITH WORK PRODUCTIVITY IN BIO-NAÏVE PATIENTS WITH ACTIVE PSORIATIC ARTHRITIS THROUGH WEEK 24 OF THE DISCOVER-2 STUDY}

J. Curtis $^{1}$, I. Mcinnes ${ }^{2}$, D. D. Gladman ${ }^{3}$, F. Yang ${ }^{4}$, S. Peterson ${ }^{4}$, P. Agarwal ${ }^{5}$ A. Kollmeier ${ }^{6}$, E. C. Hsia ${ }^{7,8}$, C. $\mathrm{Han}^{7}$, M. Shawi ${ }^{4}$, W. Tillett ${ }^{9}$, P. J. Mease ${ }^{10}$, P. Rahman ${ }^{11}$. ${ }^{1}$ University of Alabama at Birmingham, Department of Medicine, Immunology and Rheumatology, Birmingham, United States of America; ${ }^{2}$ University of Glasgow, Institute of Infection, Immunity and Inflammation, Glasgow, United Kingdom; ${ }^{3}$ Toronto Western Hospital, Centre for Prognosis in the Rheumatic Diseases, Toronto, Canada; ${ }^{4}$ Janssen Global Services, LLC, Immunology, Horsham, United States of America; ${ }^{5}$ Janssen Research \& Development, LLC, Biostatistics, Spring House, United States of America; ${ }^{6}$ Janssen Research \& Development, LLC, Immunology, San Diego, United States of America; ${ }^{7}$ Janssen Research \& Development, Immunology, Spring House, United States of America; ${ }^{8}$ University of Pennsylvania School of Medicine, Rheumatology
Philadelphia, United States of America; ${ }^{9}$ Royal National Hospital for Rheumatic Diseases, Department of Pharmacy \& Pharmacology, Centre for Therapeutic Innovation, Bath, United Kingdom; ${ }^{10}$ Swedish Medical Center/Providence St. Joseph Health and University of Washington, Rheumatology Research, Seattle, United States of America; ${ }^{11}$ Memorial University of Newfoundland, Craig L Dobbin Genetics Research Centre, St. John's, Canada

Background: Psoriatic arthritis (PsA), a chronic inflammatory disease characterized by peripheral arthritis, axial inflammation, dactylitis, enthesitis \& skin/nail psoriasis, causes impaired physical function, disability \& loss of work productivity Objectives: Evaluate associations between PsA clinical characteristics \& outcomes including fatigue \& work productivity using Work Productivity \& Activity Impairment Questionnaire: PSA (WPAI-PsA).

Methods: The Phase 3 DISCOVER-2 trial assessed guselkumab (GUS), an anti-IL-23p19 subunit monoclonal antibody, in bio-naïve adults with active PSA (swollen joint count [SJC] $\geq 5$ \& tender joint count $[T J C] \geq 5$, C-reactive protein $[\mathrm{CRP}] \geq 0.6 \mathrm{mg} / \mathrm{dL}$ ) despite standard therapies. ${ }^{1}$ Patients (Pts) were randomized 1:1:1 to GUS $100 \mathrm{mg}$ Q4W; GUS $100 \mathrm{mg}$ at W0, W4, then Q8W; or placebo (PBO). WPAI-PsA assesses PsA-related work time missed (absenteeism), impairmen while working (presenteeism), productivity loss (absenteeism+presenteeism), \& daily activity during the previous week. Spearman correlation testing evaluated relationships between pt demographics \& disease characteristics of PsA \& WPAI domain scores based on observed values at baseline. Univariate linear regression assessed associations between WPAI \& these variables based on observed data at W0 \& at W24. Variables with $p<0.10$ were included in a multivariate analysis employing a mixed-effects model for repeated measures, controlling for all other variables; resulting $\mathrm{p}$-values $<0.05$ were considered statistically significant. Results: As reported elsewhere, ${ }^{2}$ least-squares mean $\%$ changes from baseline at W24 were -3.8/-19.5/-20.0/-20.5 for GUS Q4W, -3.1/-19.4/-19.7/-21.5 for GUS Q8W, \& -3.5/-10.2/-10.9/-10.3 for PBO for absenteeism, presenteeism, absenteeism+presenteeism, \& daily activity impairment, respectively. Among 738 pts, WPAI domain scores were moderately to strongly correlated (ie, $\geq 0.4$ ) with pt-reported pain (0-10 visual analog scale), physical function (Health Assessment Questionnaire Disability Index [HAQ-DI]), fatigue (Functional Assessment of Chronic Illness Therapy-Fatigue [FACIT-F] scale) \& 36-Item Short Form Health Survey (SF-36) Physical Component Summary (PCS) score, but weakly correlated with other variables (Figure 1). Based on univariate analyses \& evaluation of collinearity between variables, attributes included in multivariate models were age, body mass index (BMI), gender, CRP, FACIT-F, pain, Psoriasis Area Severity Index (PASI), TJC, SJC enthesitis \& dactylitis. In final model, CRP, FACIT-F, \& pain were statistically significantly associated with all WPAI domains (Table 1). Presence of enthesitis \& higher PASI score were significantly associated with higher loss of work productivity \& activity outside work.

Conclusion: In PsA pts, extra-articular symptoms, fatigue, pain \& elevated CRP were significantly associated with WPAI-assessed work \& activity impairment. Treating all major clinical manifestations of PsA is needed to help pts improve work \& activity impairment. GUS effectively treats all major clinical manifestations ${ }^{1} \&$ improves work \& activity impairment in PsA. ${ }^{2}$

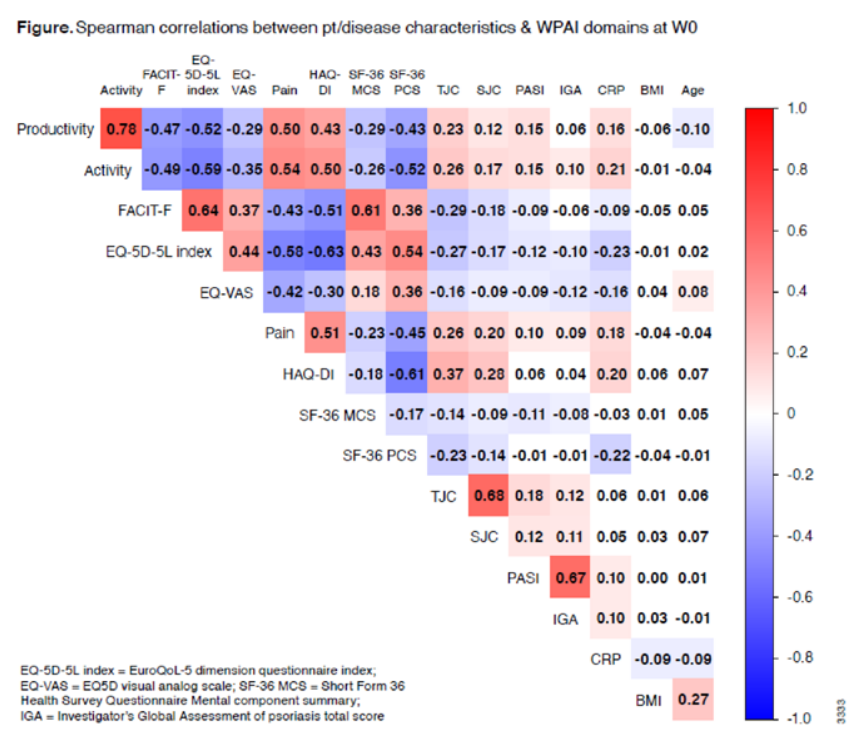

REFERENCES:

[1] Mease P. Lancet 2020;395:1126-36.

[2] Curtis J. ACR 2020; Poster 0332. 